\title{
Development of Longer-Lasting Insect Repellence Cellulosic Based Curtain Fabrics
}

\author{
Afaf Farag Shahba ${ }^{1 *}$, Osama Halawa ${ }^{1}$, Mohamed Ragaei ${ }^{2}$, Mohamed Hashem ${ }^{3}$ \\ ${ }^{1}$ Department of Spinning, Weaving and Knitting Engineering, Faculty of Applied Arts, Helwan University, Helwan, Egypt; \\ ${ }^{2}$ Department of Pests and Plant Protection, National Research Centre, Cairo, Egypt; ${ }^{3}$ Textile Division, National Research Centre, \\ Cairo, Egypt. \\ E-mail: afaffarag1999@yahoo.com
}

Received October $6^{\text {th }}$, 2010; revised February $9^{\text {th }}$, 2011; accepted February $23^{\text {rd }}, 2011$.

\begin{abstract}
Long lasting insect repellent curtain fabrics were developed and characterized. Different types of fabrics which are generally used in manufacture of curtain fabrics were functionalized with monochlorotriazenyl $\beta$-cyclodextrin (MCT$\beta-C D)$ then treated with different concentration from permethrin to impart the fabric insect repellent properties. These fabrics were $100 \%$ cotton, cotton/viscose, cotton/linen, polyester/cotton, polyester/linen and polyester/viscose blend fabrics. The treated curtain fabrics were evaluated for insect repellent retention capacity against mosquitoes. The repellent efficacy comprises the determination of percent mosquitoes repelled, knockdown and killed (mortality) resulting from exposed mosquitoes to the treated fabric. Results obtained shows that, the insect repellent retention capacity of curtain fabrics functionalized with $R-\beta-C D$ then treated with permethrin depends on the amount of $\beta$-CD moieties on the curtain fabrics, type of fabric and permethrin concentration. Higher action is obtained when the fabric was functionalized with $100 \mathrm{~g} / \mathrm{L}, M C T-\beta-C D$ in alkaline medium followed by treatment with $15 \mathrm{~g} / \mathrm{L}$ permethrin. The results show also that, curtain fabric made of cotton/linen shows highest mosquitoes repellent retention capacity and highest resistance against washing compared with $100 \%$ cotton or cotton/viscose or polyester based curtain fabrics. The insect repellent treatment of curtain fabrics did not adversely affect the tensile strength or drapability index of curtain fabrics.
\end{abstract}

Keywords: Cotton, Curtain Fabrics, $\beta$-Cyclodextrin, Linen, Insect Repellent Fabric, Polyester, Viscose

\section{Introduction}

Among the increasing number of arthropod-borne diseases, only few are preventable by vaccines. There is no effective vaccine against Malaria, the only way to avoid it remains avoiding mosquitos' bites [1-4]. For this reason, personal protective measures against biting arthropods and arthropod borne diseases constitute the first line of defense. A major advance in the protection of highrisk personnel (e.g. outdoor workers, travelers, and soldiers) is the development of insects repellent clothing, tents, and netting.

Pyrethrum is a $100 \%$ natural and environment friendly insecticide. Pyrethrum is derived from the dried flowers of Chrysanthemum Cinerariaefolium. The name given to the active insecticidal components of the dried flowers is "Pyrethrins". The Pyrethrum flower provides a highly effective protection against mosquitoes, carriers of killer diseases such as malaria and yellow fever. The physio- logical action of pyrethrins is to inhibit the mosquitoes from biting and causes repellence, immobilisation, paralysis and death [5]. Although the extract of the plant were already used as insecticide, the structure was first published by Staudinger and Ruzicka [6]. Pyrethrins are neurotoxins that attack the nervous systems of all insects. When present in amounts not fatal to insects, they still appear to have an insect repellent effect. Pyrethrins are gradually replacing organophosphates and organochlorides as the pesticide of first choice.

The pyrethrins are a pair of natural organic compounds that have potent insecticidal activity. Pyrethrin I and pyrethrin II are structurally related esters with a cyclopropane core. The chemical structure of pyrethrins is the basis for a variety of synthetic insecticides called pyrethroids such as bifenthrin, permethrin and cypermethrin [7]. The chemical structure of permethrin is as follows:

Permethrin has the following outstanding properties [7-9]: 
<smiles>CC1(C)[C@H](C=C(Cl)Cl)[C@H]1C(=O)OCc1cccc(Oc2ccccc2)c1</smiles>

Permethrin: 3-Phenoxybenzyl (1RS)-cis,trans-3-(2,2-dichlorovinyl)-2,2-dimethylcyclopropanecarboxylate.

1) Rapid Action,

2) Low Mammalian Toxicity (No other insecticide can claim such a long record of proven safety towards humans and warm blooded animals,

3) Broad Spectrum of Activity;

4) Environment friendly: degraded by the combination of sunlight and air.

Curtain fabrics are considered one of the most important upholstery fabrics. Usually, curtain fabrics are used to cover glass and ventilator windows to prevent (or reduce) direct sunlight and consequently reduce heat results thereof. In addition to this, curtains are also used to impart aesthetic fashion. Generally, curtain fabrics are divided into two types, heavy fabric (or opaque) and light fabric (or semi-transparent). Cotton, polyester, linen and their blends are frequently used in manufacture of curtain fabrics.

Cyclodextrins compounds are novel environmentally save complexing agents which are used to encapsulate fragrances compounds [10-14]. These are crystalline toxicologically harmless compound and having a circular, conical conformation, where the height is about $7.8 \AA$ and the cavity's inner diameter is between 4.7 and $8.3 \AA$ wide [10].

A fascinating property of the cyclodextrins is their ability to incorporate other organic compounds into their cavity, both in the solid state and in solution [10]. The application of cyclodextrins to the textile industries has become more interesting, for example in dye stabilization [11-16], laser dyes [17], printing [17], colour-changing compositions [11-14,18], long-lasting perfumed cotton $[14,19]$ and the new auxiliaries [11-14,19-21].

Entrepreneurs continue seeking new ideas which can give them a competitive advantage. In textiles industry, one approach is based on finishes which maximize the added value and friendly with the environment. Therefore, the aim of this work is to develop and characterize curtain fabrics having long lasting insect repellent properties. In this regards, six different type of fabrics generally used in manufacture of curtain fabrics were selected. These fabrics are $100 \%$ cotton, cotton/viscose, cotton/linen and cotton/polyester, polyester/linen and polyester/viscose blend fabrics. All these fabric were separately functionalized with monochlorotriazenyl $\beta$-cyclodextrin ( $\mathrm{R}-\beta$-CD) then treated with different concentration insect repellent agent (permethrin). The effect of permethrin concentration, degree of fabric modification with $\beta$-CD, kind of curtain material and its construction on the characteristic properties of the curtain fabric were investigated. These properties include, fabric retained tensile strength, drapability and durability of insect repellent function against several washing cycle. The influence of insect repellent finished curtain fabrics on mosquitoes (type Aedes aegypti) which include the percent repellence, knockdown and mortality was evaluated.

\section{Experimental}

\subsection{Fabrics Specification}

Two kind of warp thread are used for manufacturing curtain fabrics, cotton and polyester, whereas, three different kind of weft thread are used, namely, cotton, viscose and linen. Hence, six different substrates having different specification are produced. Table 1 summarizes the specifications of these fabrics.

\subsection{Weaving Machine Specification}

Electronic Jakard weaving machine type Picanol, Model Gama 1995 has been use in fabric manufacturing. The machine operates with 550 picks/min and the numbers of Jakard shinkels used were 3072.

\subsection{Chemical Used}

Monochlorotriazinyl- $\beta$-cyclodextrin referred here as reactive $\beta$-cyclodextrin (R- $\beta$-CD) was provided by Waker Chemie GmbH, Germany. Sodium hydroxide, acetic acid and sodium carbonate were of laboratory grade chemicals. Egyptol ${ }^{\circledR}$ (non-ionic wetting agent based on ethylene oxide condensate) and Espycon ${ }^{\circledR}$ (anionic wetting agent), commercial permethrin were of technical grade.

\subsection{Fabric Preparation}

After waving the fabrics were subjected to mild scouring on winch machine using recipe currently used by the industry described as follows: All fabrics were treated in winch machine with an aqueous solution containing $\mathrm{NaOH}(6 \mathrm{~g} / \mathrm{L})$, Egyptol ${ }^{\circledR}(2 \mathrm{~g} / \mathrm{L})$ and Espycon ${ }^{\circledR}(1 \mathrm{~g} / \mathrm{L})$ using material to liquor ratio (LR) $1: 20$ at $95^{\circ} \mathrm{C}$ for 30 min. The samples were then washed several times with boiling water and cold water and finally dried at ambient conditions

\subsection{Modification of Curtain Fabric with Reactive Cyclodextrin (R-CD) and Permethrin}

Scoured curtain fabrics were cut into pieces with a dimension of $40 \times 40 \mathrm{~cm}$. Reaction of these fabrics with $\mathrm{R}-\beta$-CD was carried out using pad-dry-cure method $[11-14,19]$ the experimental technique was adopted as follows: The fabric was padded in two dips and two nips 
Table 1. Fabric specifications.

\begin{tabular}{lcccccc}
\hline Substrate No & I & II & III & IV & V \\
\hline Type of warp yarn & Cotton & Cotton & Cotton & Polyester & Polyester & Polyester \\
Type of weft yarn & Cotton & Viscose & Linen & Cotton & Viscose & Linen \\
Number of warp yarn/cm & 48 & 48 & 48 & 72 & 72 & 28 \\
Number of weft yarn/cm & 28 & 28 & 28 & 28 & 17 & 17 \\
Warp yarn count (Tex) & 14.5 & 14.5 & 14.5 & 37 & 37 & 37 \\
Weft yarn count (Tex) & 37 & 37 & 36.6 & 35.5 & 35.5 \\
Repeat of warp & 36.6 & 36.6 & 28 & 35.5 \\
Reading set Slaying reading (dent/cm) & 24 & 24 & 24 & 9 & 9 \\
\hline
\end{tabular}

in an aqueous solution containing R- $\beta$-CD $(0-150 \mathrm{~g} / \mathrm{L})$ and sodium carbonate $(30 \mathrm{~g} / \mathrm{L})$ then squeezed to a wet pick-up of about $100 \%$. The cotton fabric was dried at $85^{\circ} \mathrm{C}$ for $5 \mathrm{~min}$ and then cured at $160^{\circ} \mathrm{C}$ for $3 \mathrm{~min}$. The fabric was washed with cold water containing $1 \%$ acetic acid followed by several washing cycles and finally dried under the normal ambient conditions.

The obtained $\beta$-CD-treated samples were separately impregnated in four different concentration from insects repellent agent $(0-20 \mathrm{~g} / \mathrm{L})$ for 20 min with occasionally steering. The fabric was then squeezed using centrifuge then dried in an air oven at $40^{\circ} \mathrm{C}$ for $30 \mathrm{~min}$ to remove excess unfixed permethrin, then dried at $50^{\circ} \mathrm{C}$ for $30 \mathrm{~min}$ then put in polyethylene bag.

\subsection{Analysis and Testing}

1) Nitrogen content of the treated fabric was determined by the Kjeldhal method [22].

2) Fabric tensile strength of was determined according to ASTM standard test method [23].

3) Fabric drapability was determined according to a reported method [24].

4) Determination of insect repellent retention capacity of the treated curtain fabric against mosquitoes (repellent efficacy) including repellent effect, knockdown effect and mortality resulting from exposed the mosquitoes to the treated fabric were measured using World Health Organization (WHO) standard cone tests method [1-3,25]. The numbers of mosquitoes repelled, knockdown and mortality were recorded after $60 \mathrm{~min}$. Mosquitoes type Aedes aegypti was used.

\section{Result and Discussion}

Monochlorotriazenyl- $\beta$-cyclodextrin (R- $\beta$-CD) reacts with cotton or linen cellulose in presence of alkaline medium to produces fabric bearing $\beta$-CD moieties. Different level from $\beta$-CD modified curtain fabrics can be produced by using different $\mathrm{R}-\beta$-CD concentration. The amount of $\beta$-CD moieties on the fabrics are monitored via determination of nitrogen content of the fabric. Cellulosic based curtain fabric bearing $\beta$-CD are allowed to react with insect repelling agents (permethrin) as inclusion into the cavities of $\beta$-cyclodextrin. It is feasible that, the treatment efficacy depend manly on the amount of $\beta$-CD moieties on the fabric and the fabric type and permethrin concentration. The effects of these two parameters are discussed below.

\subsection{Relation between the Amounts of $\beta$-CD Moieties on Cotton Based Curtain Fabric and its Insect Repellent Retention Capacity}

Table 2 shows the effect of permethrin concentration and the fabric content from $\beta$-CD moieties (expressed as nitrogen content) on amount of mosquitoes repellence, knockdown and mortality. It should be noted here that, zero nitrogen content represent those fabric did not functionalized with $\mathrm{R}-\beta$-CD whereas zero permethrin concentration represent those fabric modified with $\beta$-CD only. It is seen from Table 2 that:

1) Unmodified $100 \%$ cotton fabric (having zero N\%) did not show any influence on the mosquitoes unless it treated with permethrin. Treatment the unmodified fabric with permethrin marginally increases the percent repellence, knockdown and mortality of mosquitoes and the maximum values were obtained at permethrin concentration equal $15 \mathrm{~g} / \mathrm{L}$.

2) At constant permethrin concentration, increasing the amount of $\beta$-CD moieties on the fabric enhances the action of the treatment against mosquitoes. This was evidenced by increasing the percent repellence, percent mosquito's knockdown and percent dead mosquitoes by increasing the nitrogen content of the fabric. Higher action of finished curtain fabric made of $100 \%$ cotton against mosquitoes is observed at nitrogen content equal to $0.43 \%$. This amount of $\beta$-CD moieties fixed on the cotton fabric was attained when using $100 \mathrm{~g} / \mathrm{L}$, MCT$\beta$-CD in finishing treatment.

3) Result of Table 2 make it clear that cotton fabrics finished with $\beta$-CD shows an improvement of their insect repellent retention capacity, especially towards mosquitoes. It is also obvious that, the repellence effect of finished curtain fabric depends mainly on its content from 
Table 2. Relation between the efficacy of treated $100 \%$ cotton fabric against mosquitoes and its content from $\beta$-CD moieties (expressed as $\mathrm{N} \%$ ) and permethrin concentration.

\begin{tabular}{|c|c|c|c|c|c|c|c|c|c|c|c|c|}
\hline \multirow{3}{*}{$\begin{array}{l}\text { Perm-ethrin conc. } \\
\text { (g/L)) }\end{array}$} & \multicolumn{4}{|c|}{ Repellence \% } & \multicolumn{4}{|c|}{ Knockdown \% } & \multicolumn{4}{|c|}{ Mortality \% } \\
\hline & \multicolumn{4}{|c|}{ N\% } & \multicolumn{4}{|c|}{ N\% } & \multicolumn{4}{|c|}{ N\% } \\
\hline & $0.0^{\text {(b) }}$ & 0.21 & 0.43 & 0.61 & 0.0 & 0.21 & 0.43 & 0.61 & 0.0 & 0.21 & 0.43 & 0.61 \\
\hline $0.0^{\text {(a) }}$ & 0 & 0 & 0 & 0 & 0 & 0 & 0 & 0 & 0 & 0 & 0 & 0 \\
\hline 5 & 5 & 15 & 30 & 25 & 0 & 5 & 10 & 20 & 0 & 0 & 0 & 0 \\
\hline 10 & 16 & 30 & 63 & 61 & 3 & 10 & 30 & 40 & 0 & 2 & 5 & 5 \\
\hline 20 & 17 & 75 & 93 & 94 & 8 & 20 & 65 & 65 & 2 & 5 & 5 & 5 \\
\hline
\end{tabular}

${ }^{\text {(a) }}$ Values at zero permethrin concentration represent the efficacy of cotton or $\beta$-CD modified cotton fabric towards mosquitoes. ${ }^{\text {(b) }}$ Values at zero N\% represent the efficacy of unmodified cotton fabric towards mosquitoes.

$\beta$-CD moieties and permethrin concentration. This clearly indicated that, apart from the interactions of the permethrin molecules with the cotton cellulose network (physical adsorption, hydrogen bonding), the $\beta$-CD molecules played a major role. Improvement of insect repellent retention capacity is attributed to the formation of an inclusion complex between permethrin molecules and the cavity of $\beta$-CD molecules on cotton fabric in addition to other interactions like hydrogen bonding and physical surface adsorption [11-13,19].

Tables 3 and $\mathbf{4}$ show the effect of permethrin concentration and the fabric content from $\beta$-CD moieties (expressed as nitrogen content) on insect repellent retention capacity of curtain fabric made of cotton/viscose and cotton/linen respectively. Results of Tables $\mathbf{3}$ and $\mathbf{4}$ are similar to that obtained with $100 \%$ cotton fabric (Table 2) and could be explained on similar basis.

\subsection{Relation between the Amounts of $\beta$-CD Moieties on Polyester Based Curtain Fabric and its Insect Repellent Retention Capacity}

Table 5 shows the effect of permethrin concentration and the fabric content from $\beta$-CD moieties (expressed as nitrogen content) on amount of mosquitoes repellence, knockdown and mortality. It should be noted here that, zero nitrogen content represent those fabric did not functionalized with $\mathrm{R}-\beta$-CD whereas zero permethrin concentration represent those fabric modified with $\beta$-CD only. It is seen from Table 2 that, Unmodified polyester/cotton fabric (having zero N\%) did not show any influence on the mosquitoes. Treatment the unmodified fabric with different concentration from permethrin increases the percent repellence, knockdown and mortality of mosquitoes and the maximum values were obtained at permethrin concentration equal $15 \mathrm{~g} / \mathrm{L}$.

Comparing these results with the results obtained with 100\% cotton fabric (Table 2) reveals that, at the same permethrin concentration, the insect repellent retention capacity of unmodified polyester/cotton fabric is greater than that observed with unmodified $100 \%$ cotton fabrics. This may be due to the similarity between the hydrophobic nature of polyester component in the fabric to the hydrophobic nature of permethrin which enhances the surface binding of permethrin onto the polyester fabric via Van der Waal force.

It is further noted from Table 5 that, at constant permethrin concentration, increasing the amount of $\beta$-CD moieties on the fabric enhances the action of the treatment against mosquitoes. This was evidenced by increasing the percent repellence, percent mosquito's knockdown and percent dead mosquitoes by increasing the nitrogen content of the fabric. Higher action of finished curtain fabric made of polyester/cotton against mosquitoes is observed at nitrogen content equal to $0.31 \%$. This amount of $\beta$-CD moieties fixed on the polyester/cotton fabric was attained when using $100 \mathrm{~g} / \mathrm{L}$, MCT- $\beta$-CD in finishing treatment.

Result of Table 5 clearly indicated that, apart from the interactions of the permethrin molecules with the polyester and cotton cellulose network (Van der Waal physical adsorption, hydrogen bonding), the $\beta$-CD molecules played a major role. Improvement of insect repellent retention capacity is attributed to the formation of an inclusion complex between permethrin molecules and the cavity of $\beta$-CD molecules onto polyester/cotton fabric.

Tables 6 and 7 show the effect of permethrin concentration and the fabric content from $\beta$-CD moieties (expressed as nitrogen content) on insect repellent retention capacity of curtain fabric made of polyester/viscose and polyester/linen respectively. Results of Tables 6 and 7 are similar to that obtained with polyester/cotton fabric (Table 5) and could be explained on similar basis.

Comparison between the results obtained in Tables 5-7 show that, at approximately similar nitrogen content and permethrin concentration, the insect repellent retention capacity of polyester based fabric fellow the order: polyester/linen > polyester/cotton > polyester/viscose fabric. 
Table 3. Relation between the efficacy of treated cotton/viscose fabric against mosquitoes and its content from $\beta$-CD moieties (expressed as $\mathrm{N} \%$ ) and permethrin concentration.

\begin{tabular}{|c|c|c|c|c|c|c|c|c|c|c|c|c|}
\hline \multirow{3}{*}{$\begin{array}{l}\text { Perm-ethrin } \\
\text { conc. (g/L)) }\end{array}$} & \multicolumn{4}{|c|}{ Repellence \% } & \multicolumn{4}{|c|}{ Knockdown \% } & \multicolumn{4}{|c|}{ Mortality \% } \\
\hline & \multicolumn{4}{|c|}{ N\% } & \multicolumn{4}{|c|}{ N\% } & \multicolumn{4}{|c|}{ N\% } \\
\hline & $0.0^{\text {(b) }}$ & 0.23 & 0.45 & 0.65 & 0.0 & 0.23 & 0.45 & 0.65 & 0.0 & 0.23 & 0.45 & 0.65 \\
\hline $0.0^{(\mathrm{a})}$ & 0 & 0 & 0 & 0 & 0 & 0 & 0 & 0 & 0 & 0 & 0 & 0 \\
\hline 5 & 5 & 20 & 50 & 55 & 0 & 3 & 15 & 37 & 0 & 0 & 0 & 0 \\
\hline 10 & 10 & 40 & 62 & 65 & 5 & 8 & 30 & 55 & 0 & 1 & 4 & 4 \\
\hline 20 & 20 & 78 & 94 & 96 & 10 & 39 & 65 & 68 & 2 & 5 & 7 & 7 \\
\hline
\end{tabular}

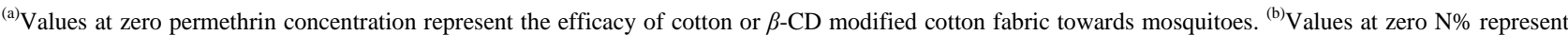
the efficacy of unmodified cotton fabric towards mosquitoes.

Table 4. Relation between the efficacy of treated cotton/linen fabric against mosquitoes and its content from $\beta$-CD moieties (expressed as $\mathrm{N} \%$ ) and permethrin concentration.

\begin{tabular}{|c|c|c|c|c|c|c|c|c|c|c|c|c|}
\hline \multirow{3}{*}{$\begin{array}{l}\text { Perm-ethrin } \\
\text { conc. (g/L) }\end{array}$} & \multicolumn{4}{|c|}{ Repellence \% } & \multicolumn{4}{|c|}{ Knockdown \% } & \multicolumn{4}{|c|}{ Mortality \% } \\
\hline & \multicolumn{4}{|c|}{ N\% } & \multicolumn{4}{|c|}{$\mathrm{N} \%$} & \multicolumn{4}{|c|}{$\mathrm{N} \%$} \\
\hline & $0.0^{(\mathrm{b})}$ & 0.27 & 0.49 & 0.67 & 0.0 & 0.27 & 0.49 & 0.67 & 0.0 & 0.27 & 0.49 & 0.67 \\
\hline $0.0^{\text {(a) }}$ & 0 & 0 & 0 & 0 & 0 & 0 & 0 & 0 & 0 & 0 & 0 & 0 \\
\hline 5 & 5 & 23 & 28 & 36 & 8 & 0 & 10 & 20 & 0 & 0 & 0 & 0 \\
\hline 10 & 19 & 35 & 77 & 86 & 11 & 10 & 30 & 40 & 0 & 4 & 4 & 8 \\
\hline 15 & 23 & 65 & 98 & 99 & 12 & 29 & 76 & 75 & 2 & 6 & 10 & 9 \\
\hline 20 & 30 & 87 & 99 & 99 & 17 & 31 & 77 & 75 & 2 & 8 & 10 & 10 \\
\hline
\end{tabular}

${ }^{\text {(a) }}$ Values at zero permethrin concentration represent the efficacy of cotton or $\beta$-CD modified cotton fabric towards mosquitoes. ${ }^{\text {(b) }}$ Values at zero N\% represent the efficacy of unmodified cotton fabric towards mosquitoes.

Table 5. Relation between the efficacy of treated polyester/cotton fabric against mosquitoes and its content from $\beta$-CD moieties (expressed as $\mathrm{N} \%$ ) and permethrin concentration.

\begin{tabular}{|c|c|c|c|c|c|c|c|c|c|c|c|c|}
\hline \multirow{3}{*}{$\begin{array}{l}\text { Perm-ethrin } \\
\text { conc. }(\mathrm{g} / \mathrm{L})\end{array}$} & \multicolumn{4}{|c|}{ Repellence \% } & \multicolumn{4}{|c|}{ Knockdown \% } & \multicolumn{4}{|c|}{ Mortality \% } \\
\hline & \multicolumn{4}{|c|}{ N\% } & \multicolumn{4}{|c|}{ N\% } & \multicolumn{4}{|c|}{ N\% } \\
\hline & $0.0^{(\mathrm{b})}$ & 0.17 & 0.27 & 0.42 & 0.0 & 0.17 & 0.27 & 0.42 & 0.0 & 0.17 & 0.27 & 0.42 \\
\hline $0.0^{(\mathrm{a})}$ & 0 & 0 & 0 & 0 & 0 & 0 & 0 & 0 & 0 & 0 & 0 & 0 \\
\hline 5 & 15 & 16 & 25 & 33 & 8 & 8 & 10 & 16 & 3 & 3 & 5 & 5 \\
\hline 10 & 25 & 26 & 29 & 65 & 12 & 10 & 22 & 31 & 4 & 3 & 5 & 5 \\
\hline 15 & 30 & 25 & 74 & 75 & 20 & 35 & 55 & 53 & 5 & 6 & 9 & 9 \\
\hline 20 & 35 & 42 & 76 & 77 & 31 & 38 & 55 & 53 & 5 & 8 & 8 & 9 \\
\hline
\end{tabular}

${ }^{\text {(a) }}$ Values at zero permethrin concentration represent the efficacy of cotton or $\beta$-CD modified cotton fabric towards mosquitoes. ${ }^{\text {b) }}$ Values at zero N\% represent the efficacy of unmodified cotton fabric towards mosquitoes.

Table 6. Relation between the efficacy of treated polyester/viscose fabric against mosquitoes and its content from $\beta$-CD moieties (expressed as $\mathrm{N} \%$ ) and permethrin concentration.

\begin{tabular}{|c|c|c|c|c|c|c|c|c|c|c|c|c|}
\hline \multirow{3}{*}{$\begin{array}{l}\text { Perm-ethrin } \\
\text { conc. (g/L) }\end{array}$} & \multicolumn{4}{|c|}{ Repellence \% } & \multicolumn{4}{|c|}{ Knockdown \% } & \multicolumn{4}{|c|}{ Mortality \% } \\
\hline & \multicolumn{4}{|c|}{$\mathrm{N} \%$} & \multicolumn{4}{|c|}{$\mathrm{N} \%$} & \multicolumn{4}{|c|}{ N\% } \\
\hline & $0.0^{\text {(b) }}$ & 0.17 & 0.29 & 0.41 & 0.0 & 0.17 & 0.29 & 0.41 & 0.0 & 0.17 & 0.29 & 0.41 \\
\hline $0.0^{(\mathrm{a})}$ & 0 & 0 & 0 & 0 & 0 & 0 & 0 & 0 & 0 & 0 & 0 & 0 \\
\hline 5 & 17 & 18 & 18 & 32 & 8 & 7 & 10 & 16 & 3 & 2 & 2 & 2 \\
\hline 10 & 27 & 25 & 43 & 69 & 12 & 15 & 22 & 31 & 4 & 3 & 3 & 4 \\
\hline 15 & 31 & 36 & 81 & 80 & 28 & 27 & 57 & 53 & 5 & 5 & 5 & 6 \\
\hline 20 & 32 & 42 & 80 & 81 & 31 & 30 & 55 & 53 & 5 & 6 & 6 & 7 \\
\hline
\end{tabular}

${ }^{(a)}$ Values at zero permethrin concentration represent the efficacy of cotton or $\beta$-CD modified cotton fabric towards mosquitoes. ${ }^{\text {(b) }}$ Values at zero N\% represent the efficacy of unmodified cotton fabric towards mosquitoes. 


\subsection{Effect of the Curtain Fabric Type on Mosquitoes Repellent Retention Capacity}

Table 8 shows the effect of fabric type on mosquitoes repellent retention capacity. It is seen that, the insect repellent retention capacity of curtain fabric treated with $\mathrm{R}-\beta$-CD followed by permethrin depends on the fabric type. Cotton based curtain fabrics $(100 \%$ cotton, cotton/viscose and cotton/linen fabrics) shows higher insect repellent retention capacity compared with polyester based curtain fabrics. Cellulose reacts more readily than polyester with $\mathrm{R}-\beta$-CD in alkaline medium. Therefore, curtain fabrics have higher cellulose content will acquire higher amount from $\beta$-CD moieties, which in turn accommodate higher permethrin molecules inside $\beta$-CD cavities.

Based on the above results (Tables 2-8), it could be emphasized that; the insect repellent retention capacity of curtain fabric treated with $\mathrm{R}-\beta$-CD followed by permethrin depends on the fabric type and directly proportion to the amount of fixed $\beta$-CD moieties on the fabric and permethrin concentration.

\subsection{Effect of Insect Repellence Treatment on Tensile Strength and Drapability of Perfumed Curtain Fabrics}

Tensile strength and drapability is the most important properties of curtain fabrics. In order to examine the ef- fect of our insects' repellence finishing treatment on the retained tensile strength and drapability of curtain fabrics, both drapability and tensile strength were evaluated before and after the treatments.

Figure 1 shows the retain tensile strength of the treated curtain fabrics under investigation. It is seen from Figure 1 that, 100\% cotton perfumed curtain fabric has retained tensile strength amounted 93\%, whereas those made from cotton/viscose and cotton/linen have retained tensile strength $85 \%$ and $94 \%$ respectively. The decreement in tensile strength of $100 \%$ cotton and cotton/linen curtain fabrics after insect repellent finishing treatment is due to the action of alkali on cellulose at higher temperature during it functionalization with $\mathrm{R}-\beta$-CD. The effect of these alkali and temperature on cotton/viscose fabric is more pronounced; therefore the retained tensile strength of cotton/viscose is much lower.

It is seen also from Figure 1 that, the retained tensile strength of polyester based curtain fabrics after insect repellent finishing fellow the order: polyester/linen > polyester/cotton > polyester/viscose. It is further observed that the polyester based curtain fabrics show higher retained tensile strength in comparison with cotton based fabrics after the treatment.

Figure 2 shows the drapability of curtain fabrics under investigation before and after insect repellent finishing treatment. It should be noted here that higher drapability

Table 7. Relation between the efficacy of treated polyester/linen fabric against mosquitoes and its content from $\beta$-CD moieties (expressed as $\mathrm{N} \%$ ) and permethrin concentration.

\begin{tabular}{|c|c|c|c|c|c|c|c|c|c|c|c|c|}
\hline \multirow{3}{*}{$\begin{array}{l}\text { Perm-ethrin } \\
\text { conc. (g/L) }\end{array}$} & \multicolumn{4}{|c|}{ Repellence \% } & \multicolumn{4}{|c|}{ Knockdown \% } & \multicolumn{4}{|c|}{ Mortality \% } \\
\hline & \multicolumn{4}{|c|}{$\mathrm{N} \%$} & \multicolumn{4}{|c|}{ N\% } & \multicolumn{4}{|c|}{$\mathrm{N} \%$} \\
\hline & $0.0^{\text {(b) }}$ & 0.20 & 0.31 & 0.42 & 0.0 & 0.20 & 0.31 & 0.42 & 0.0 & 0.20 & 0.31 & 0.42 \\
\hline $0.0^{(\mathrm{a})}$ & 0 & 0 & 0 & 0 & 0 & 0 & 0 & 0 & 0 & 0 & 0 & 0 \\
\hline 5 & 20 & 22 & 28 & 32 & 8 & 8 & 10 & 16 & 3 & 3 & 4 & 4 \\
\hline 10 & 29 & 31 & 39 & 69 & 16 & 15 & 22 & 31 & 4 & 4 & 3 & 5 \\
\hline 15 & 38 & 38 & 87 & 88 & 22 & 27 & 65 & 63 & 5 & 5 & 6 & 6 \\
\hline 20 & 39 & 42 & 85 & 90 & 22 & 29 & 66 & 63 & 5 & 6 & 5 & 7 \\
\hline
\end{tabular}

${ }^{\text {(a) }}$ Values at zero permethrin concentration represent the efficacy of cotton or $\beta$-CD modified cotton fabric towards mosquitoes. ${ }^{\text {(b) }}$ Values at zero N\% represent the efficacy of unmodified cotton fabric towards mosquitoes.

Table 8. Effect of the curtain fabric type on mosquitoes repellent retention capacity.

\begin{tabular}{|c|c|c|c|c|}
\hline Type of curtain fabric & N\% & Repellence \% & Knockdown \% & Mortality \% \\
\hline $100 \%$ cotton fabric & 0.43 & 93 & 64 & 5 \\
\hline Cotton/viscose & 0.45 & 95 & 67 & 7 \\
\hline Cotton/linen & 0.49 & 98 & 79 & 10 \\
\hline Polyester/cotton & 0.27 & 74 & 55 & 2 \\
\hline Polyester/viscose & 0.29 & 81 & 57 & 3 \\
\hline polyester/linen & 0.31 & 87 & 65 & 6 \\
\hline
\end{tabular}

Conditions Used: Curtain fabrics were modified with $\beta$-CD then treated with permethrin as described in the experimental part. Permethrin concentration, 15 g/L. 


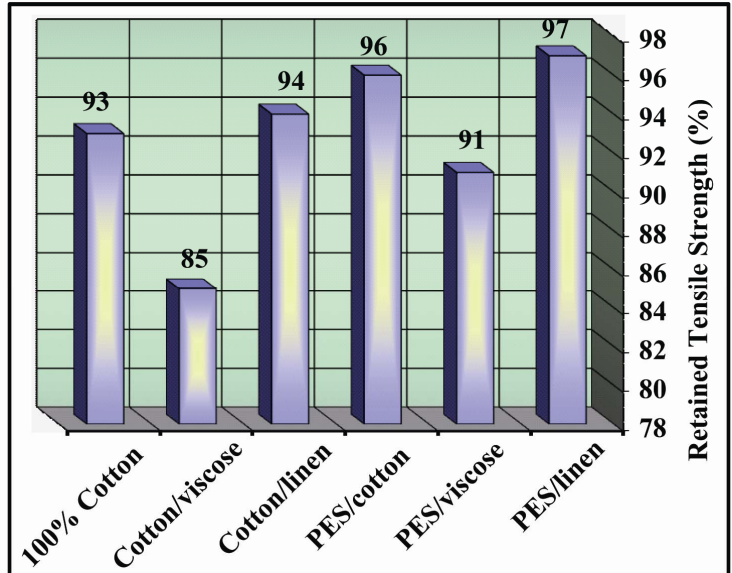

Figure 1. Retained tensile strength of curtain fabricafter treatment with $\beta$-CD and permethrin. Conditions used: Reaction of curtain fabrics with $\beta$-CD then treatment with permethrin are described in the experimental part. Nitrogen content of the treated fabric are listed in Table 8.
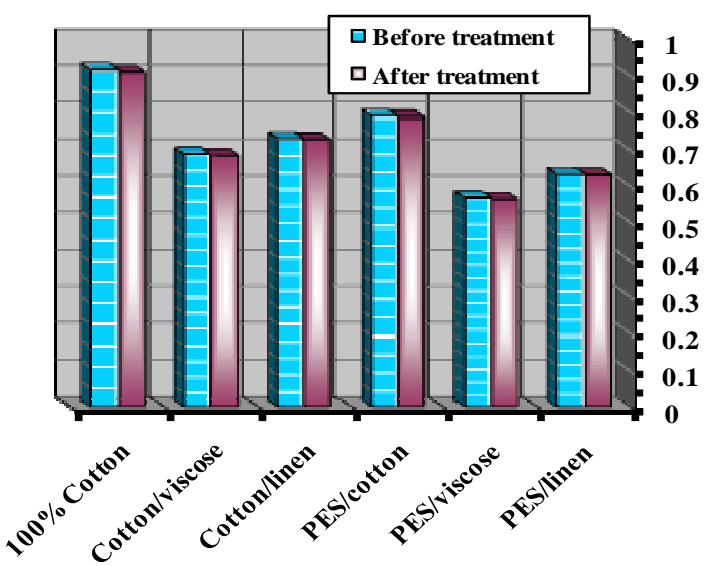

Figure 2. Drapability index of curtain fabrics before and after treatment with $\beta$-CD and permethrin. Conditions used: Reaction of curtain fabrics with $\beta$-CD then treatment with permethrin are described in the experimental part. Nitrogen content of the treated fabric are listed in Table 8. Permethrin concentration $(15 \mathrm{~g} / \mathrm{L})$.

index indicates higher stiff fabric and vice versa.

It is seen from Figure 2 that, curtain fabrics shows a variable drapability index depending on the type of fibres used in manufacturing the fabrics. The drapability indexes of curtain fabrics before insect repellent finishing treatment have the following order:

$100 \%$ cotton $>$ PES/cotton $>$ cotton/linen $>$ cotton/viscose $>$ PES/linen $>$ PES/viscose.

Figure 2 shows also that values of drapability index of curtain fabrics before and insect repellent finishing treatment are very comparable and have the same order.

Results of Figures $\mathbf{1}$ and $\mathbf{2}$ make it clear that, long lasting treatment of curtain fabrics under investigation did not adversely affect on the drapability properties or tensile strength of the fabrics.

\subsection{Durability of Insect Repellent Retention Capacity against Washing}

Curtain fabrics under investigation were functionalized with $100 \mathrm{~g} / \mathrm{L}$ from $\mathrm{R}-\beta$-CD in alkaline medium then treated with $15 \mathrm{~g} / \mathrm{L}$ permethrin as explained in the experimental part. These fabrics were subjected to mild 15 washing cycle. Each cycle comprises washing the treated fabric with warm water at $50^{\circ} \mathrm{C}$ under stirring for $15 \mathrm{~min}$ then dried. The fabrics were then evaluated for insect repellent retention capacity after every five washing cycles. This was carried out in order to investigate the resistance of the insect repellent finishing treatment to repeated washing. The insect repellent retention capacity includes the percent repelled mosquitoes, percent mosquitoes knockdown, and percent mosquitoes killed (mortality). Results obtained are represented in Figures 3-5. It should be noted here that, zero washing cycle represent the insect repellent retention capacity of curtain fabrics directly after preparation and before washing.

It is seen from Figures 3-5 that:

1) The percent mosquitoes repellence of $100 \%$ cotton fabric gradually decreases as the number of washing cycles increase. After 15 washing cycles the percent repellence reached to approximately $45 \%$. Similar trends are observed for curtained fabric made of cotton/viscose and cotton/linen fabric where the percent mosquitoes repellence reached to $50 \%$ and $60 \%$ respectively.

口 $100 \%$ Cotton $\square$ Cotton/viscose $\square$ Cotton/linen $\square$ PES/cotton $\square$ PES/viscose $\square$ PES/linen

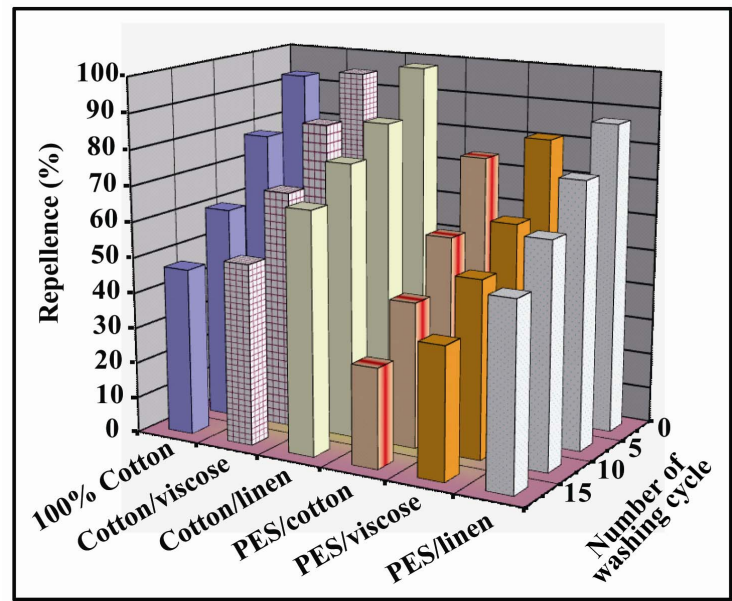

Figure 3. Effect of repeated laundry on the \% repellence of the permethrin treated curtain fabric. Conditions used: Reaction of curtain fabrics with $\beta$-CD then treatment with permethrin is described in the experimental part. Nitrogen content of the treated fabric is listed in Table 8. Permethrin concentration, $15 \mathrm{~g} / \mathrm{L}$. 


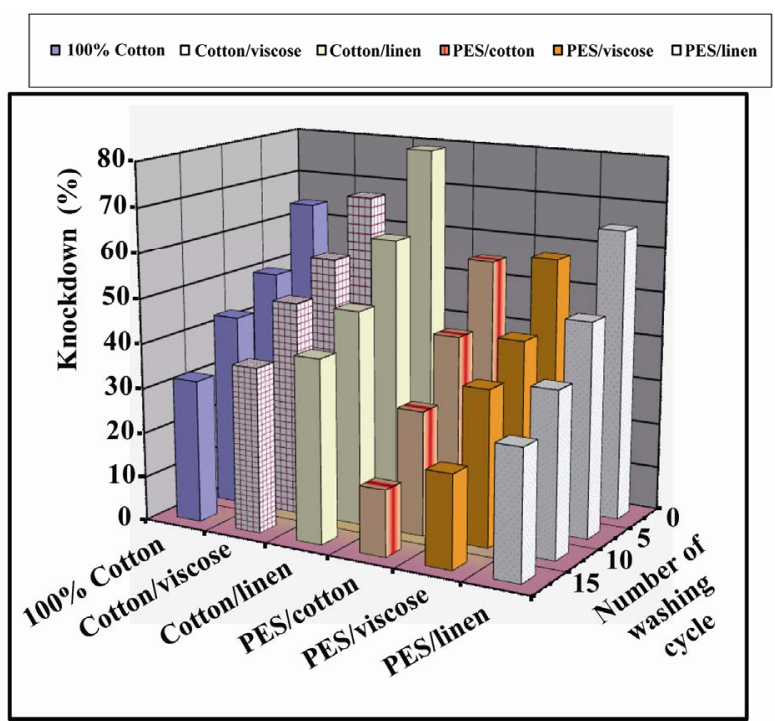

Figure 4. Effect of repeated laundry on the \% knockdown of the permethrin treated curtain fabric. Conditions used: Reaction of curtain fabrics with $\beta$-CD then treatment with permethrin are described in the experimental part. Nitrogen content of the treated fabric are listed in Table 8. Permethrin concentration $(15 \mathrm{~g} / \mathrm{L})$.

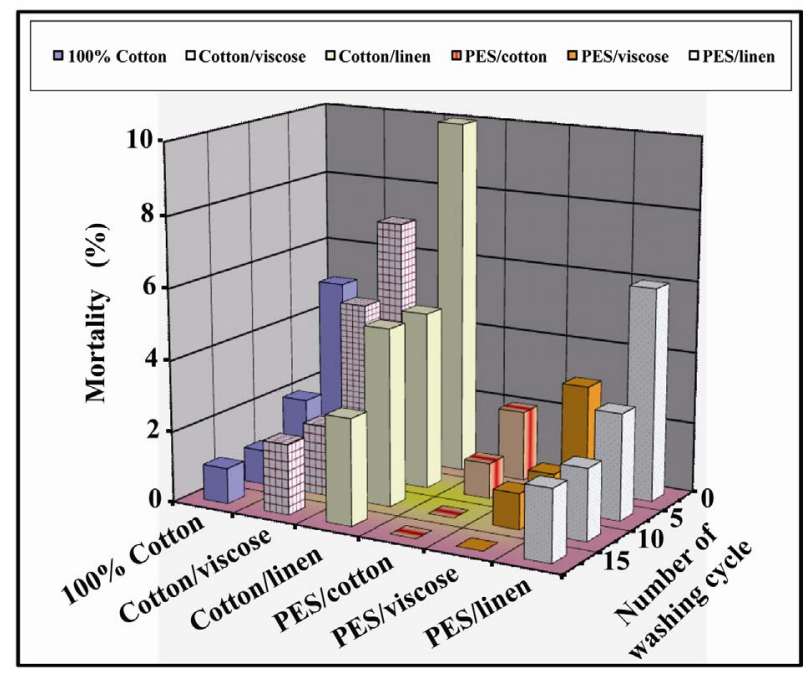

Figure 5. Effect of repeated laundry on the \% mortality of the permethrin treated curtain fabric. Conditions used: Reaction of curtain fabrics with $\beta$-CD then treatment with permethrin are described in the experimental part. Nitrogen content of the treated fabric are listed in Table 8. Permethrin concentration (15 g/L).

2) For polyester based curtain fabrics, the percent mosquitoes repellence also decreases by increases the number of washing cycle. After 15 washing cycle, the percent repellence of polyester/cotton, polyester/viscose and polyester/linen fabric was amounted $20 \%, 30 \%$, and $40 \%$ respectively.
3) Results of Figure 3 make it clear also that; durability of the percent repellence against washing depends on the type of curtain fabric and its content from $\beta$-CD moieties. The higher fabric content from $\beta$-CD moieties the higher residual repellence after repeated washing.

4) Figures 4,5 show the change in percent mosquitoes knockdown, and percent mosquitoes killed (mortality) respectively, after repeated washing. Results obtained are represented in Figures 4,5 are similar to those obtained in Figure 3 and could be explained on similar basis.

\section{Conclusions}

The insect repellent retention capacity of curtain fabrics functionalized with R- $\beta$-CD then treated with permethrin depends on the amount of $\beta$-CD moieties on the curtain fabrics (expressed as nitrogen content), type of fabric and permethrin concentration. At the same permethrin concentration, increasing the amount of $\beta$-CD moieties on the fabric enhances the repellent retention capacity action against mosquitoes. Higher action is observed at permthrin concentration equal to $15 \mathrm{~g} / \mathrm{L}$ and nitrogen content equal to $0.43 \%$. This amount of $\beta$-CD moieties fixed on the cotton fabric was attained when using $100 \mathrm{~g} / \mathrm{L}$, MCT- $\beta$-CD during finishing treatment. Results also show that, curtain fabric made of cotton/linen shows highest mosquitoes repellent retention capacity and highest resistance against washing compared with $100 \%$ cotton or cotton/viscose. Polyester based curtain fabrics shows lower insect repellent retention capacity compared with cellulosic based fabric. The insect repellent treatment of curtain fabrics under investigation did not adversely affect the tensile strength or drapability index of curtain fabrics.

\section{REFERENCES}

[1] WHO, "Insecticide Resistance and Vector Control: 17th Report of the Expert Committee on Insecticides," WHO Technical Report Series, Vol. 443, 1970, pp. 47-56.

[2] WHO, "Vectors of Diseases: Hazards and Risks for Travelers-Part I,” WER 25, 2001, pp. 189-194.

[3] WHO, "Vectors of Diseases: Hazards and Risks for Travelers-Part II,” WER 26, 2001, pp. 201-203.

[4] WHO, "Environmental Health Criteria 94-Permethrin; International Program on Chemical Safety," World Health Organization, Geneva, 1990, pp. 26-33.

[5] D. Young and S. Evans, "Safety and Efficacy of DEET and Permethrin in the Prevention of Arthropod Attack," Military Medicine, Vol. 163, No. 5, 1998, pp. 1-7.

[6] H. Staudinger and L. Ruzicka, "Über Die Wirksamen Bestandteile Des Dalmatinischen Insektenpulvers,” Helvetica Chimica Acta, Vol. 7, 1924, p. 177. doi:10.1002/hlca.19240070124

[7] J. M. G. Wainaina, "Pyrethrum Flowers-Production in 
Africa,” In: E. C. John and B. Q. Gary, Eds., Pyrethrum Flowers, Oxford University Press, Oxford, 1995, pp. 49-54.

[8] C. D. S. Tomlin, “The Pesticide Manual, A World Compendium,” 14th Edition, British Crop Production Council, Alton, 2006.

[9] R. H. Ian, “Aquatic Organisms and Pyrethroids," Pesticide Science, Vol. 27, No. 4, 1989, pp. 429-457.

[10] F. Vogtle, "Supramolecular Chemistry: An Introduction, Chapter 4, Bio-Organic Model Compounds,” John Wiley and Sons, New York, 1993.

[11] H. J. Buschmann, D. Knittel and E. Schollmeyer, ”Cyclodextrins and Dextrins as New Auxiliaries in Dyeing," Melliand Textilberichte, Vol. 72, No. 12, 1991, 1012-1014.

[12] H. J. Buschmann, D. Knittel and E. Schollmeyer, "Resin finishing of Cotton in the Presence of Cyclodextrins for Depositing Fragrances,” Melliand Textilberichte, Vol. 72, No. 3, 1991, pp. E75-E76.

[13] H. J. Buschmann and E. Schollmeyer, “Textile Material Finished with Polymeric Cyclodextrins, and Method for the Production Thereof," Journal of Inclusion Phenomena and Molecular Recognition in Chemistry, Vol. 14, 1991, p. 91.

[14] H. J. Buschmann, D. Knittel and E. Schollmeyer, "Textile Materialien Mit Cyclodextrinen, Textile Materials with Cyclodextrins,” Melliand Textilberichte, Vol. 82, No. 5, 2001, pp. 368-370.

[15] B. Martel, M. Morcellet, D. Ruffin, F. Vinet and M. Weltrowski, "Capture and Controlled Release of Fragrances by CD Finished Textiles," Journal of Inclusion Phenomena and Macrocyclic Chemistry, Vol. 44, 2003, pp. 439-442. doi:10.1023/A:1023028105012

[16] B. Martel, M. Morcellet, D. Ruffin, F. Vinet and M. Weltrowski, "Polycarboxylic Acids as Crosslinking Agents for Grafting Cyclodextrins onto Cotton and Wool Fabrics:
Study of the Process Parameters," Journal of Applied Polymer Science, Vol. 83, No. 7, 2002, pp. 1449-1456. doi:10.1002/app.2306

[17] S. Okano, "Printing of Cellulosic Fibers and Blends," Japanese Kokai Tokkyo Koho, Vol. 114, 1978, p. 987.

[18] H. J. Buschmann, D. Knittel and E. Schollmeyer, 'Possibilities of the Use of Cyclodextrin Dye Complexes in Dyeing Processes,” Textilveredlung, Vol. 31, No. 5-6, 1996, pp. 115-117.

[19] H. J. Buschmann, U. Dente, D. Knittel and E. Schollmeyer, "Processing Methods for Permanent Fixing of Cyclodextrin Derivatives on Textile Surfaces," Textilveredlung, Vol. 32, No. 1-2, 1997, pp. 33-39.

[20] D. Knittel, H. J. Buschmann and E. Schollmeyer, "Finishing of Natural and Synthetic Fibres by Fixation of Cyclodextrin Derivatives,” Textilveredlung, Vol. 26, 1991, p. 92.

[21] A. Hebeish and Z. H. El-Hilw, "Chemical Finishing of Cotton Using Reactive Cyclodextrin,” Coloration Technology, Vol. 117, No. 2, 2001, pp. 104-110. doi:10.1111/j.1478-4408.2001.tb00343.x

[22] A. Vogel, "Elementary Practical Inorganic Chemistry Part 3, Quantitative Inorganic Analysis,” 2nd Edition, Longman, London, 1975.

[23] ASTM, "Breaking Load and Elongation of Textile Fabric”, D-1682-94, 1994.

[24] ISO 9073-9, "Test Methods for Non-Woven, Part 9. Evaluation of Drapability including Drape Coefficient," British Standards Institution, 2006.

[25] N. Kapoor and M. A. Anari, "Laboratory Evaluation of Synthetic Pyrethroid Treated Cotton Fabric against Mosquitoes and other Domestic Pests,” Journal of Tropical Medicine and Parasitology, Vol. 26, 2003, pp. 20-25. 